\title{
Stability Analysis of Large Scale Networks of Autono- mous Work Systems with Delays
}

\author{
H.R. Karimi ${ }^{1}$, S. Dashkovskiy ${ }^{2}$, N.A. Duffie ${ }^{3}$ \\ ${ }^{1}$ Faculty of Technology and Science, University of Agder, Grimstad, \\ Norway (e-mail: hamid.r.karimi@uia.no) \\ ${ }^{2}$ Centre for Industrial Mathematics, University of Bremen, Bremen, \\ Germany (e-mail: hrkarimi@math.uni-bremen.de) \\ ${ }^{3}$ Department of Mechanical Engineering, University of Wisconsin- \\ Madison, Madison, WI, USA
}

\begin{abstract}
This paper considers the problem of stability analysis for a class of production networks of autonomous work systems with delays in the capacity changes. The system under consideration does not share information between work systems and the work systems adjust capacity with the objective of maintaining a desired amount of local work in progress (WIP). Attention is focused to derive explicit sufficient delay-dependent stability conditions for the network using properties of matrix norm. Finally, numerical results are provided to demonstrate the proposed approach.
\end{abstract}

\section{INTRODUCTION}

Production networks are emerging as a new type of cooperation between and within companies, requiring new techniques and methods for their operation and management [1]. Coordination of resource use is a key challenge in achieving short delivery times and delivery time reliability. These networks can exhibit unfavourable dynamic behaviour as individual organizations respond to variations in orders in the absence of sufficient communication and collaboration, leading to recommendations that supply chains should be globally rather than locally controlled and that information sharing should be extensive [2]-[3]. However, the dynamic and structural complexity of these emerging networks inhibits collection of the information necessary for centralized planning and control, and decentralized coordination must be provided by logistic processes with autonomous capabilities [4]. 
A production network with several autonomous work systems is depicted in Figure 1. The behaviour of such a network is affected by external and internal order flows, planning, internal disturbances, and the control laws used locally in the work systems to adjust resources for processing orders [5]. In prior work, sharing of capacity information between work systems has been modelled [6] along with the benefits of alternative control laws and reducing delay in capacity changes [7][8]. Several authors have described both linear and nonlinear dynamical models for control of variables such as inventory levels and work in progress (WIP), including the use of pipeline flow concepts to represent lead times and production delays ([9], [10]). Delivery reliability and delivery time have established themselves as equivalent buying criteria alongside product quality and price (see [1], [11]). High delivery reliability and short delivery times for companies demand high schedule reliability and short throughput times in production. In order to manufacture economically under such conditions, it is necessary to minimise WIP levels in production and utilise operational resources in the best possible way.



Fig. 1. Production network consisting of a group of autonomous work systems.

Production Planning and Control (PPC) has become more challenging as manufacturing companies adapt to a fast changing market [12]-[14]. Current PPC methods often do not deal with unplanned orders and other types of turbulence in a satisfactory manner [15]. Assumptions such as infinite capacity and fixed lead time are often made, leading to a static view of the production system may not be valid because WIP affects lead time and performance, while capacity is finite and varies both according to plan and due to unplanned disturbances such as equipment breakdowns, worker illness, market changes etc. Understanding the dynamic na- 
ture of production systems requires new approaches for the design of PPC based on company's logistics [16]. The controllers implicitly interact to adjust capacity to eliminate backlog as the system maintains its planned WIP level [15]. A discrete closed-loop PPC model was developed and analyzed by Duffie and Falu [17] in which two discrete controllers, one for backlog and one for WIP, with different periods between adjustments of work input and capacity, respectively, were selected and evaluated using transfer function analysis and time-response simulation. A second architecture for continuous WIP control and discrete backlog control, with delay capacity adjustment, was developed and analyzed by Ratering and Duffie for cases of high and low WIP [18].

On the other hand, delay differential systems are assuming an increasingly important role in many disciplines like economic, mathematics, science, and engineering. For instance, in economic systems, delays appear in a natural way since decisions and effects are separated by some time interval. The delay effects problem on the stability of systems is a problem of recurring interest since the delay presence may induce complex and undesired behaviors (oscillation, instability, bad performance) for the schemes [19]-[23]. Over the past few decades, discrete-time systems with time-delay have received little attention compared with its continuous-time counterpart ([24]-[27]). The stability of time-delay systems is a fundamental problem because of its importance in the analysis of such systems. With regard to the stability analysis issue, Verriest and Ivanov in [28] studied the sufficient conditions for the asymptotic stability of the discrete-time state delayed systems by using an algebraic matrix inequality approach. The basic method for stability analysis is the direct Lyapunov method, and by this method, strong results have been obtained. But finding Lyapunov functions for nonautonomous delay difference systems is usually a difficult task. In contrast, many methods different from Lyapunov functions have been successfully applied to establish stability results for difference equations with delay, for example, [29]-[31]. Recently, in [32] a computational method was presented using Haar wavelets to determine the piecewise constant feedback controls for a finite-time linear optimal control problem of a time-varying state-delayed system.

In this paper, we contribute to the problem of stability analysis for a class of production networks of autonomous work systems with delays in the capacity changes. The system under consideration does not share information between work systems and the work systems adjust capacity with the objective of maintaining a desired amount of WIP. Attention is focused to derive explicit sufficient delay-dependent stability conditions for the network using properties of matrix norm. Finally, numerical results are provided to demonstrate the proposed approach.

\section{Model of autonomous work systems}

A linear discrete-time dynamic approach for modeling the flow of orders into, out of, and between work systems was chosen because it promotes straightforward calculation of fundamental dynamic properties such as characteristic times and 
damping. Assume that there are $N$ work systems in a production network, as shown in Figure 1, and that vector $i(n T)$ is the rate at which orders are input to the $N$ work systems from sources external to the production network, which is constant over time $n T \leq t<(n+1) T$ where $n=0,1,2, \cdots$ and $T$ is a time period between capacity adjustments (for example, 1 shop-calendar day [scd]). The total orders that have been input to the work systems up to time $(k+1) T$ then can be represented as the vector [5]

$$
w_{i}((n+1) T)=w_{i}(n T)+T\left(i(n T)+R(n T)^{T} C_{a}(n T)\right)
$$

where vector $c_{a}(n T)$ is the rate at which orders are output from the $N$ work systems during time $n T \leq t<(n+1) T$ (the actual capacity of each work system) and $R$ is a matrix in which element approximates the fraction of the flow out of work system $j$ that flows into work system $k$.

The total number of orders that have been output by the work systems up to time $n T \leq t<(n+1) T$ can be represented by the vector

$$
w_{o}((n+1) T)=w_{o}(n T)+T c_{a}(n T)
$$

while the rate at which orders are output from the network during time $n T \leq t<(n+1) T$ is

$$
o(n T)=R_{o}(n T) C_{a}(n T)
$$

where $R_{o}(n T)$ is a diagonal matrix in which non-zero diagonal elements represents the fraction of orders flowing out of work systems that flow out of the network during time $n T \leq t<(n+1) T . R_{o}(n T)$ is assumed to be constant during this period, and

$$
R_{o_{i i}}(n T)+\sum_{\substack{j=1 \\ j \neq i}}^{N} R_{o_{i j}}(n T)=1
$$

$R(n T)$ and $R_{o}(n T)$ represent the structure of order flow in the network. The WIP in the work systems is

$$
\operatorname{wip}_{a}(n T)=w_{i}(n T)-w_{o}(n T)+w_{d}(n T)
$$

where $w_{d}(n T)$ represents local work disturbance, such as rush order, that affect the work system. Furthermore, the actual capacity of each work system depends on three components as follows:

$$
c_{a}(n T)=c_{p}(n T)+c_{m}((n-d) T)-c_{d}(n T)
$$

where $c_{d}(n T)$ represents local capacity disturbances such as equipment failures, $c_{p}(n T)$ denotes planned capacities of the work systems and $c_{m}(n T)$ represents local capacity adjustments to maintain the WIP in each work system in the vicinity of the planned levels wip $p_{p}(n T)$ using gain $k_{c}$ and is described in the form of

$$
c_{m}(n T)=k_{c}\left(w_{i p}(n T)-w_{i p}(n T)\right)
$$

It is assumed that a delay $d T$ exists in the capacity changes $c_{m}(n T)$ for logistic reasons such as operator work rules. In this network, the work systems do not 
share information regarding the expected physical flow of orders between them. A capacity plan is required for each work system. For constants $R(n T)$ and $R_{o}(n T)$, the transfer functions relating $\operatorname{wip}_{a}(z)$ and $c_{a}(z)$ to the inputs $i(z), w_{d}(z)$, $\operatorname{wip}_{p}(z), c_{p}(z)$ and $c_{d}(z)$ are

$$
\begin{aligned}
& \operatorname{wip}_{a}(z)=\left(\left(I-z^{-1}\right) I+k_{c} T\left(I-R^{T}\right) z^{-(d+1)}\right)^{-1}\left(T z^{-1} i(z)+\left(I-z^{-1}\right) w_{d}(z)\right. \\
& \left.\quad+k_{c} T\left(I-R^{T}\right) z^{-(d+1)} \operatorname{wip}_{p}(z)-T\left(I-R^{T}\right) z^{-1} c_{p}(z)+T\left(I-R^{T}\right) z^{-1} c_{d}(z)\right) \\
& \operatorname{wip}_{a}(z)=\left(\left(I-z^{-1}\right) I+k_{c} T\left(I-R^{T}\right) z^{-(d+1)}\right)^{-1}\left(T z^{-1} i(z)+\left(I-z^{-1}\right) w_{d}(z)\right. \\
& \left.\quad+k_{c} T\left(I-R^{T}\right) z^{-(d+1)} \operatorname{wip}_{p}(z)-T\left(I-R^{T}\right) z^{-1} c_{p}(z)+T\left(I-R^{T}\right) z^{-1} c_{d}(z)\right)
\end{aligned}
$$

Our purpose is to investigate the stability of the network (1) respect to the delay parameter and the controller gain which is characterize by the roots of

$$
\operatorname{det}\left(\left(I-z^{-1}\right) I-A z^{-(d+1)}\right)=0
$$

with $A:=-k_{c} T\left(I-R^{T}\right)$.

\section{Stability analysis}

In this section, sufficient conditions for the stability of the network (1) respect to the delay parameter and the controller gain are proposed using characteristic equation.

The characteristic equation (3) can be represented in the form of

$$
\operatorname{det}\left(A+I z^{d}-I z^{(d+1)}\right)=0
$$

and (4) is corresponding to the characteristic equation of the following system

$$
x_{n}=x_{n-1}+A x_{n-d-1}
$$

Levitskaya in [30] established that (5) is asymptotically stable if and only if any eigenvalue of the matrix $A$ lies inside the oval of the complex plane bounded by a curve

$$
\Gamma=\left\{z \in C: z=2 i \sin \frac{\varphi}{2 d+1} e^{i \varphi},|\varphi| \leq \frac{\pi}{2}\right\} .
$$

Remark 1. Let $\lambda_{i}$ be eigenvalues of the matrix $A=-k_{c} T\left(I-R^{T}\right)$. The equation (5) is asymptotically stable if and only if

$$
\left|\lambda_{i}\right|<2 \sin \frac{\pi}{2(2 d+1)}
$$

Theorem 1. If the system (5) is asymptotically stable, then all eigenvalues of A lie inside the unit disk.

Proof. It is sufficient to consider the stability ovals (6) and to remark that $|2 \sin (\pi / 2(2 \mathrm{~d}+1))| \leq 1$ for $\mathrm{k}>1$. 
In the sequel, we will obtain the necessary and sufficient condition in terms of the eigenvalues location of the matrix $A$ for the asymptotic stability of the equation (5).

Lemma 1. [29] If $\sum_{i=1}^{k}\left\|A_{i}\right\|<1$, then the linear system $x_{n}=\sum_{i=1}^{k} A_{i} x_{n-i}$ is asymptotically stable.

Theorem 2. If

$$
\|A+I\|+d\|A\|^{2}<1
$$

then (4) is asymptotically stable.

Proof. The equation (5) is rewritten as

$$
\begin{aligned}
x_{n} & =(A+I) x_{n-1}-A\left(x_{n-1}-x_{n-d-1}\right) \\
& =(A+I) x_{n-1}-A \sum_{i=1}^{d}\left(x_{n-i}-x_{n-i-1}\right) \\
& =(A+I) x_{n-1}-A \sum_{i=1}^{d} A x_{n-i-d-1}
\end{aligned}
$$

According to Lemma 1, from (8) we conclude (7).

Now, we introduce an additional stability condition for (5) depending on whether the delay $d$ is odd or even.

\section{Theorem 3. If}

$$
\left\|I+(-1)^{d} A\right\|+d\|A\|(2+\|A\|)<1
$$

then (4) is asymptotically stable.

Proof. If $d$ is even the equation (5) is rewritten as

$$
\begin{aligned}
x_{n} & =(A+I) x_{n-1}-A\left(x_{n-1}-x_{n-d-1}\right) \\
& =(A+I) x_{n-1}-A \sum_{i=1}^{d}(-1)^{i+1}\left(2 I x_{n-i-1}+A x_{n-k-i}\right)
\end{aligned}
$$

and if $d$ is odd we have

$$
\begin{aligned}
x_{n} & =(I-A) x_{n-1}+A\left(x_{n-1}-x_{n-d-1}\right) \\
& =(I-A) x_{n-1}+A \sum_{i=1}^{d}(-1)^{i+1}\left(2 I x_{n-i-1}+A x_{n-k-i}\right)
\end{aligned}
$$

Similar to the proof of Theorem 2, the inequality (9) is concluded.

\section{Numerical Results}

Consider the case of a supplier of components to the automotive industry and for which production data documents orders flowing between five work systems over a 162-day period. These work systems and the order-flow structure over this period is illustrated in Figure 2. In this network, all order flows are unidirectional; 
therefore, the fundamental dynamic properties of capacity adjustment in the individual work systems are independent. Then, the internal flow of orders is approximated using the following matrix [5],

$$
R=\left[\begin{array}{ccccc}
0 & 106 / 341 & 235 / 341 & 0 & 0 \\
0 & 0 & 0 & 188 / 401 & 204 / 401 \\
0 & 0 & 0 & 100 / 236 & 129 / 236 \\
0 & 0 & 0 & 0 & 268 / 295 \\
0 & 0 & 0 & 0 & 0
\end{array}\right]
$$

in which element $R_{i j}$ is the total number of orders that went from work system $i$ to work system $j$ divided by the total number of orders that left work system $i$.

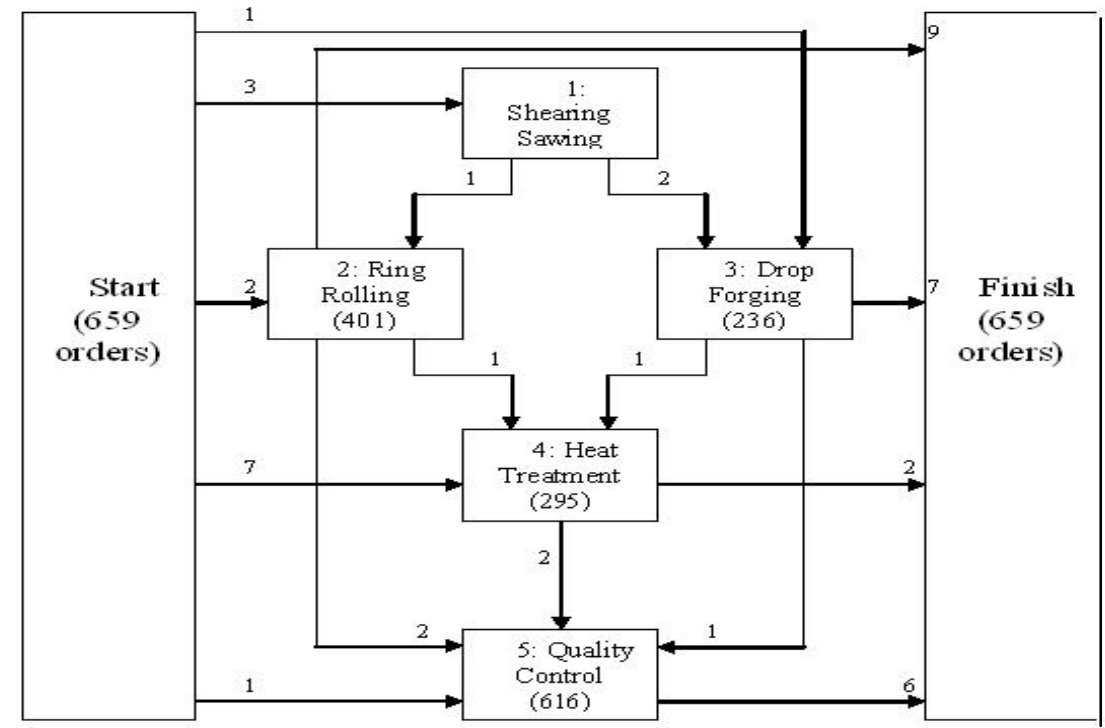

Fig. 2. A production network consisting of five work systems.

Consider the sampling time $T=1 \mathrm{scd}$. It is clear that the condition in Lemma 1 cannot be applied. Applying all of the Theorems derived, the conditions of maximum controller gain for the asymptotic stability of the network are shown in Table 1. The result from Table 1 guarantees the asymptotic stability of system under consideration. 
Table 1

Controller gain $k_{c}$ w.r.t. $d$.

\begin{tabular}{|c|c|r|r|}
\hline & Theorem 1 & Theorem 2 & Theorem 3 \\
\hline$d_{2}=1$ & 1 & 0.8500 & 0.8650 \\
\hline$d_{2}=2$ & 0.6180 & 0.6250 & 0.6850 \\
\hline$d_{2}=3$ & 0.4450 & 0.4750 & 0.4875 \\
\hline$d_{2}=4$ & 0.3473 & 0.3845 & 0.3950 \\
\hline
\end{tabular}

\section{Conclusion}

The problem of stability analysis for a class of production networks of autonomous work systems with delays in the capacity changes was investigated in this paper. The system under consideration does not share information between work systems and the work systems adjust capacity with the objective of maintaining a desired amount of local work in progress (WIP). In terms of properties of matrix norm some explicit sufficient delay-dependent stability conditions were derived for the network. Finally, numerical results were provided to demonstrate the proposed approach.

\section{Acknowledgment}

This research has been funded by the German Research Foundation (DFG) as part of the Collaborative Research Center 637 'Autonomous Cooperating Logistic Processes: A Paradigm Shift and its Limitations’ (SFB 637).

\section{Appendix}

|.| is any matrix norm which satisfies the following conditions:

(I) $\|A\| \geq 0$, and $\|A\|=0$ if and only if $A=0$,

(II) for each $c \in R,\|c A\|=|c|\|A\|$,

(III) $\|A+B\| \leq\|A\|+\|B\|$,

(IV) $\|A B\| \leq\|A\| .\|B\|$ for all $(m \times m)$ matrices $A, B$.

In addition, matrix norm should be concordant with the vector norm \|\|$_{*}$, that is,

$$
\|A x\|_{*} \leq\|A\| \cdot\|x\|_{*}
$$


for all $x \in \Re^{m}$ and any $(m \times m)$ matrix $A$. For real $(m \times m)$ matrix $A$, we define,

as usual, $\|A\|_{1}=\max _{1 \leq j \leq m} \sum_{i=1}^{m}\left|a_{i j}\right|$ and $\|A\|_{\infty}=\max _{1 \leq i \leq m} \sum_{j=1}^{m}\left|a_{i j}\right|$.

\section{References}

[1] Wiendahl H.-P., Lutz S., 'Production in networks.' Annals of the CIRP, vol. 52, no. 2, pp. 573-586, 2002.

[2] Helo P., 'Dynamic modelling of surge effect and capacity limitation in supply chains.' Int. J. Production Research, vol. 38, no. 17, pp. 4521-4533, 2000.

[3] Huang G.Q., Lau J.S.K., Mak K.L., 'The impacts of sharing production information on supply chain dynamics: A review of the literature.' Int. J. Production Research, vol. 41, no. 7, pp. 1483-1517, 2003.

[4] Monostori, L., Csaji, B. Cs., Kadar, B., 'Adaptation and learning in distributed production control,' Annals of the CIRP, vol. 53, no. 1, pp. 349-352, 2004.

[5] Duffie N.A., Roy D., Shi L., 'Dynamic modelling of production networks of autonomous work systems with local capacity control' CIRP AnnalsManufacturing Technology, vol. 57, pp. 463-466, 2008.

[6] Kim J.-H., Duffie N., 'Performance of coupled closed-loop workstation capacity controls in a multi-workstation production system.' Annals of the CIRP, vol. 55, no. 1, pp. 449-452, 2006.

[7] Kim J.-H., Duffie N., 'Design and analysis of closed-loop capacity control for a multi-workstation production system.' Annals of the CIRP, vol. 54, no. 1, pp. 455-458, 2005.

[8] Nyhuis P., Cieminski G., Fischer A., 'Applying simulation and analytical models for logistic performance prediction.' Annals of the CIRP, vol. 54, no. 1, pp. 417-422, 2005.

[9] John S., Naim M.M., Towill D.R., 'Dynamic analysis of a WIP compensated decision support system.' Int. J. Manufacturing System Design, vol. 1, no. 4, pp. 283-297, 1994.

[10] Bai, S. X., Gershwin, S. B., 'Scheduling manufacturing systems with workin-process inventory control: Multi-part-type systems,' Int. J. Production Research, vol. 32, no. 2, pp. 365-385, 1994.

[11] Towill D.R., Evans G.N., Cheema P., 'Analysis and design of an adaptive minimum reasonable inventory control system.' J. Production Planning and Control, vol. 8, no. 6, pp. 545-577, 1997.

[12] Ratering, A., Duffie, N., 'Design and analysis of a closed-loop singleworkstation PPC system.' Annals of CIRP, vol. 52, no. 1, pp. 355-358, 2003.

[13] Scholz-Reiter B., Freitag M., de Beer C., Jagalski T., 'Modelling dynamics of autonomous logistic processes: Discrete-event versus continuous approaches.' CIRP Annals - Manufacturing Technology, vol. 54, no. 1, pp. 413-416, 2005.

[14] Wiendahl H.-P., Breithaupt J.-W., 'Automatic production control applying control theory.' Int. J. Production Economics, vol. 63, no. 1, pp. 33-46, 2000.

[15] Kim, J.-H., Duffie, N. A, 'Backlog control for a closed loop PPC system.' ClRP Annals, vol. 53, no. 1, pp. 357-360, 2004. 
[16] Wiendahl, H.-H., Roth, N., Westkamper, E., 'Logistical positioning in a turbulent environment,' Annals of the CIRP, vol. 5111, pp. 383-386, 2002.

[17] Duffie N., Falu I., 'Control-theoretic analysis of a closed-loop PPC system.' Annals of the CIRP, vol. 5111, pp. 379-382, 2002.

[18] Ratering A., Duffie N., 'Design and analysis of a closed-loop singleworkstation PPC system.' Annals of the CIRP, vol. 5211, pp. 355-358, 2003.

[19] Han Q.L., 'Robust stability of uncertain delay-differential systems of neutral type.' Automatica, vol. 38, no. 4, pp. 719-723, 2002.

[20] Fridman, E., 'New Lyapunov-Krasovskii functionals for stability of linear retarded and neutral type systems.' Systems \& Control Letters, vol. 43, pp. 309319, 2001.

[21] He Y., Wang Q.G., Lin C., Wu M., 'Delay-range-dependent stability for systems with time-varying delay.' Automatica, vol. 43, pp. 371-376, 2007.

[22] Park, P., 'A delay-dependent stability criterion for systems with uncertain time-invariant delays.' IEEE Trans. Automatic Control, vol. 44, pp. 876-877, 1999.

[23] Karimi, H.R., 'Observer-based mixed $H_{2} / H_{\infty}$ control design for linear systems with time-varying delays: An LMI approach.' Int. J. Control, Autom., and Systems, vol. 6, no. 1, pp. 1-14, 2008.

[24] Boukas E.K., Liu Z.K., 'Robust $H_{\infty}$ control of discrete-time Markovian jump linear systems with mode-dependent time-delays.' IEEE Trans. Automat. Control, vol. 46, pp. 1918-1924, 2001.

[25] Wang Z., Huang B., Unbehauen H., 'Robust $H_{\infty}$ observer design of linear state delayed systems with parametric uncertainty: the discrete-time case.' Automatica, vol. 35, pp. 1161-1167, 1999.

[26] Gao, H., Lam, J., Xie, L.H., Wang, C.H., 'New approach to mixed $H_{2} / H_{\infty}$ filtering for polytopic discrete-time systems' IEEE Trans. on Signal Processing, vol. 53, no. 8, pp. 3183-3192, 2005.

[27] Gao, H., Lam, J., Wang, Z., 'Discrete bilinear stochastic systems with timevarying delay: Stability analysis and control synthesis' Chaos, Solitons and Fractals, vol. 34, no. 2, pp.394-404, 2007.

[28] Verriest, E., and Ivanov, A.F., 'Robust stability of delay-difference equations.' Proc. 34th Conf. on Decision and Control, pp. 386-391, New Orleans, USA, 1995.

[29] Kipnis M., and Komissarova D., 'Stability of a delay differrence system.' Advances in Difference Equations, vol. 2006, Article ID 31409, pp. 1-9.

[30] Levitskaya I.S., 'A note on the stability oval for $x_{n+1}=x_{n}+A x_{n-k}$.' J. Difference Equations and Applications, vol. 11, no. 8, pp. 701-705, 2005.

[31] Kipnis M. and Nigmatulin R.M., 'Stability of trinomial linear difference equations with two delays,' Automation and Remote Control, vol. 65, no. 11, pp. 1710-1723, 2004.

[32] Karimi H.R., 'A computational method for optimal control problem of timevarying state-delayed systems by Haar wavelets' International Journal of Computer Mathematics, vol. 83, no. 2, pp. 235-246, 2006. 